\title{
VIOLÊNCIA DOMÉSTICA DURANTE A PANDEMIA DO COVID-19
} DOMESTIC VIOLENCE DURING THE PANDEMIC OF COVID-19

\author{
ARTIGO DE REVISÃO recebido 22/02/2021, aceito em 09/03/2021 \\ Como citar: Yoshino F, Caselli VM, Muñoz-Gianvecchio D, Dontos AC. Violência Doméstica na Pandemia. \\ Persp Med Legal Pericias Med. 2021; 6: e210304 \\ https://dx.doi.org/10.47005/060104
}

Fernanda Yoshino ${ }^{(1)}$

Currículo Lattes: 4772721065400762 - ID ORCID: 0000-0001-8545-7680

Victória Marsura Caselli (1)

Currículo Lattes: 5303068266553943 - ID ORCID: 0000-0003-09405015

Daniele Muñoz Gianvecchio (2)

Currículo Lattes: 3804734858598400 - ID ORCID: 0000-0001-61886130

Athanase Christos Dontos (3)

Currículo Lattes: 9892516587201021 - ID ORCID: 0000-0002-8017-331X

\author{
(1) Faculdade de Medicina da Universidade Santo Amaro, Departamento de Medicina Legal, \\ São Paulo - SP, Brasil. (autor principal) \\ (2) Faculdade de Ciências Médicas da Santa Casa de São Paulo e da Faculdade de Medicina da USP, \\ Departamento de Medicinal Legal, São Paulo - SP, Brasil. (orientador) \\ (3) Faculdade de Medicina da Universidade Santo Amaro, Departamento de Medicina Legal, \\ São Paulo - SP, Brasil. (orientador) \\ e-mail: vick_caselli@hotmail.com
}

\section{RESUMO}

INTRODUÇÃO: A violência doméstica é a ação realizada por um integrante de uma família que esteja em relação de poder com outro, prejudicando sua integridade. O aumento de casos de violência se deve à mudança na dinâmica da família, como consequência do isolamento social. OBJETIVO: analisar se a pandemia impactou no número de casos de violência doméstica. Materiais e métodos: Revisão da literatura nas bases cientificas do PubCovid-19, Scielo e Órgãos de Saúde Pública com os descritores: "Covid-19", "violência doméstica", "distanciamento social", "abuso" e "pandemia", onde foram encontrados 89 artigos nas línguas inglesa e portuguesa. RESULTADO: Observa-se que o aumento da violência se dá em razão do isolamento social pelo aumento do convívio com agressores, além da falta de liberdade para procurar ajuda. DISCUSSÃO: durante o período de pandemia, alguns fatores propiciaram o aumento da violência doméstica: as instituições de ensino e trabalho encontram-se fechadas, multitarefa, crise econômica e irritabilidade pelas restrições impostas aumentam comportamentos agressivos. Além disso, a dependência, seja social ou financeira, muitas vezes tornam as vítimas mais vulneráveis. CONCLUSÃO: o confinamento dificulta o alcance das vítimas de 
violência doméstica. Assim, é proposto a garantia de atendimento em tempo integral através de canais de comunicação e estabelecimento de medidas para organização da rotina, fortalecimento de laços e transformações positivas nas dinâmicas familiares.

Palavras-chave: violência, violência doméstica, violência familiar, isolamento social, pandemia, covid-19.

\section{ABSTRACT}

INTRODUCTION: Domestic violence is the action performed by a member of a family who is in a power relationship with another, harming their integrity. The increase in cases of violence is due to the change in the dynamics of the family, because of social isolation. OBJECTIVE to analyze whether the pandemic impacted the number of cases of domestic violence. Materials and methods: Review of existing literature, where 89 articles were found in English and Portuguese within scientific databases PubCovid-19, Scielo and Public Health Agencies using descriptors: "Covid-19", "domestic violence", "social distancing", "abuse" and "pandemic". RESULT: It is observed that the increase in violence is due to social isolation as a result of increased interaction with aggressors, in addition to the lack of freedom to seek help. DISCUSSION: during the pandemic period, some factors have led to the increase in domestic violence: educational and work institutions are closed, multitasking, economic crisis and irritability by the restrictions imposed, increase aggressive behaviors. In addition, dependency, whether social or financial, often makes victims more vulnerable. CONCLUSION: confinement makes it difficult for victims of domestic violence to reach for help. Thus, it is proposed to guarantee full-time care through communication channels and establishment of measures for routine organization, strengthening of ties and positive transformations in family dynamics.

Keywords: violence, domestic violence, family violence, social isolation, pandemic, covid-19.

\section{INTRODUÇÃO}

A violência doméstica ou também conhecida como violência intrafamiliar é definida por "toda ação ou omissão que prejudique o bem-estar, a integridade física, psicológica ou a liberdade e o direito ao pleno desenvolvimento de um membro da família. Pode ser cometida dentro ou fora de casa, por qualquer integrante da família que esteja em relação de poder com a pessoa agredida. Inclui também as pessoas que estão exercendo a função de pai ou mãe, mesmo sem laços de sangue" (1). E enfrentar a violência doméstica é uma prioridade de saúde pública, uma vez que é relatada em todos os países e em todos os grupos socioeconômicos (2).

Com a pandemia do novo corona vírus SARS-CoV-2, causador da COVID-19, ainda sem tratamento efetivo, as estratégias de enfrentamento do problema têm sido baseadas em medidas de cuidados individuais, fazendo o uso de máscara de proteção e lavagem regular das mãos, visando a prevenção da transmissão via respiratória e por contato, como o distanciamento social (3).

Um risco elevado de violência doméstica está associado a esta e outras medidas de redução da infecção. Os motivos citados incluem isolamento social, exposição à estressores econômicos e psicológicos, aumento de mecanismos de enfrentamento negativo (como o uso indevido de álcool e drogas), incapacidade de acessar os mecanismos de apoio usuais ou escapar de famílias abusivas devido a medidas restritivas (4).

Assim, as repercussões do distanciamento social nas relações interpessoais expressam certa preocupação com as evidências no aumento da violência doméstica, onde o lar, frequentemente, se torna um lugar de medo e abuso (5). O objetivo desse trabalho é analisar como a pandemia do SARS-CoV-2 impactou no número de casos de violência doméstica.

\section{MATERIAL E MÉTODO}

Trata-se de uma revisão integrativa da literatura sobre o impacto da violência doméstica durante o período da pandemia da COVID-19. Foram revisados artigos na língua portuguesa e inglesa, tendo como bases cientificas: PubCovid-19, a qual está indexada na Biblioteca Nacional de Medicina dos Estados Unidos (PubMed), Scielo, além de 
Órgãos de Saúde Pública e livros a respeito da violência contra vulneráveis. Foram usados os seguintes descritores: "Covid-10", "violência doméstica", "distanciamento social", "abuso" e "pandemia". Resultante do último ano, foram encontrados 89 artigos e destes, foram selecionados 25 artigos que se mostraram pertinentes à pesquisa proposta.

\section{RESULTADOS}

A violência doméstica passou a ser mais discutida no meio científico a partir da década de 80 , onde a Constituição Federal e o Estatuto da Criança e do Adolescente começaram a agir de maneira eficaz, garantindo a proteção desses grupos de maior vulnerabilidade através do reconhecimento de seus direitos e de programas específicos para essa problemática (6). Apesar disso, a violência contra as mulheres sempre existiu no nosso país. Isso se dá pelo fato do Brasil se inserir em uma cultura patriarcal, estabelecendo uma relação hierárquica entre os gêneros, causando sensação de poderio masculino (7). Em um estudo de base populacional realizado com amostra representativa de 15 anos ou mais mostrou que $43 \%$ das brasileiras declaram ter sofrido violência praticada por um homem na vida (8). Outro grupo que, devido sua fragilidade, é alvo de violência doméstica é o de idosos. De 1991 a 2015, a população com mais de 60 anos no Brasil, dobrou. E é uma parcela da população composta por pessoas que tendem a ter uma maior suscetibilidade a problemas de saúde, abandono, dependência econômica e internação em instituições de longa permanência $(9,10)$.

Em busca da cura do mal que afeta a sociedade globalizada atualmente, o corona vírus, a comunidade cientifica visa o distanciamento social, o isolamento social e até mesmo o lockdown bloqueio total de contato - para reduzir a velocidade de transmissão viral, dos casos de óbitos, oportunizar tempo para melhores planos terapêuticos e prevenir um colapso no sistema de saúde. Porém, essas medidas podem trazer importantes impactos sociais e econômicos, como o desemprego e a violência (11).

No Brasil, de acordo com dados do 180 (Central de Atendimento à Mulher), do Ministério da Mulher, Família e Direitos Humanos, houve um aumento de $17 \%$ de ligações em forma de denúncia a respeito da violência contra a mulher no mês de março de 2020, quando foi recomendado o distanciamento social pela primeira vez (tabela 1). Dados do Ministério Público do Estado do Rio de Janeiro, apontaram para um aumento em $50 \%$ nos casos de violência doméstica no primeiro final de semana após o decreto de isolamento social, sendo a maioria dos casos com envolvimento de violência contra mulheres. Situações semelhantes foram registradas nos estados do Paraná, Ceará, Pernambuco e São Paulo (12).

De acordo com organizações veiculadas à mídia, houve um aumento de violência contra crianças e adolescentes na pandemia, tanto física (tabela 2) como sexual (tabela 3) sendo representada em 7,4\% no Distrito Federal; 8,5\% no Paraná; 32\% em Pernambuco e $73 \%$ no Rio Grande do Sul (13).

Além disso, a SaferNet, uma associação civil do direito privado, com atuação nacional voltada para promoção e defesa dos direitos humanos na internet no Brasil, em parceria com o Comitê Nacional de Enfrentamento à Violência Sexual contra Crianças e Adolescentes, registrou um aumento de $108 \%$ nas reclamações sobre pornografia infantil durante o mês de abril de 2020, no Brasil.

\begin{tabular}{|l|c|c|c|c|}
\hline DATA & LIGAÇōES - 180 & LIGAÇ̃̃ES-100 & TOTAL & DENUNCIAS \\
\hline MÉDIA 1-16/03 & 3045 & 7116 & 10161 & 829 \\
\hline MÉDIA 17-25/03 & 3303 & 7160 & 10463 & 978 \\
\hline \% COMPARATIVO & $8,47 \%$ & $0,62 \%$ & $2,97 \%$ & $17.97 \%$ \\
\hline
\end{tabular}

Tab. 1: Segundo a Ouvidoria Nacional de Direitos Humanos (ONDH), do Ministério da Mulher, da Família e dos Direitos Humanos (MMFDH), a média diária entre os dias 1 e 16 de março foi de 3.045 ligações recebidas e 829 denúncias registradas, contra 3.303 ligações recebidas e 978 denúncias registradas entre 17 e 25 deste mês.

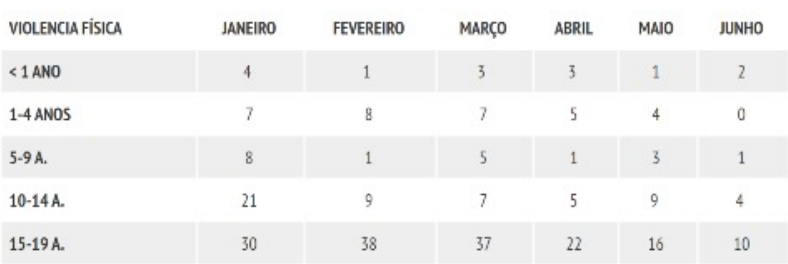

Tab. 2: Número de notificações de violência física, segundo faixa etária e ano. Distrito Federal, 2020. 


\begin{tabular}{|l|c|c|c|c|c|c|}
\hline VIOLENCIA SEXUAL & IANEIRO & FEVEREIRO & MARŞO & ABRIL & MAIO & JUNHO \\
\hline <1 ANO & 2 & 2 & 1 & 1 & 0 & 0 \\
\hline 1-4 ANOS & 21 & 13 & 11 & 6 & 7 & 2 \\
\hline 5-9 A. & 39 & 11 & 15 & 8 & 10 & 6 \\
\hline $\mathbf{1 0 - 1 4 A .}$ & 31 & 30 & 39 & 27 & 14 & 10 \\
\hline 15-19 A. & 23 & 27 & 28 & 14 & 12 & 16 \\
\hline
\end{tabular}

Tab. 3: Número de notificações de violência sexual, segundo faixa etária e ano. Distrito Federal, 2020.

\section{DISCUSSÃO}

Apesar dos números se mostrarem superiores, as vítimas da violência doméstica na pandemia continuam as mesmas - crianças e adolescentes, mulheres e idosos.

Visando à contenção da COVID-19, as instituições de ensino como creches públicas e privadas, escolas e universidades se mantiveram fechadas; assim como os comércios e empresas que estão atuando no teletrabalho. Esse modelo de sociedade cria uma dinâmica das crianças e dos adolescentes com sua família, uma vez que ambos os lados precisam recriar o ambiente de estudo e de trabalho, e conciliar o mesmo com as relações interpessoais dentro do lar. A multitarefa dos pais, a crise econômica e sanitária e social em que o mundo enfrenta, e até mesmo a irritabilidade dos mais novos pela restrição de mobilidade, aumenta a incidência de comportamentos agressivos e de irritabilidade (14).

Referente às mulheres, a violência que mais acomete esse grupo durante o período de pandemia tem sido a violência conjugal. De acordo com o Fundo de População das Nações Unidas, a elevação da vulnerabilidade das mesmas se dá pelo aumento de tensão entre a família e, principalmente, pelo impacto econômico gerado por essa situação (15). Em grande parcela da população brasileira, a responsabilidade de garantia de recursos financeiros é masculina e, na atual conjuntura, a dificuldade de manutenção do sustento familiar, junto da limitação de acesso à alimentação e outros itens essenciais, se tornam catalisadores de conflitos (16).

Em relação a terceira idade, boa parte dos componentes desse grupo do nosso país são dependentes de pensões e aposentadorias para o seu sustento e, muitas vezes, do sustendo de suas gerações dependentes que residem no mesmo domicílio (17). Porém, muitos deles são abandonados pelo fato de os familiares não conseguirem garantir condições de acolher o idoso durante a velhice. Há aqueles que residem em instituições de longa permanência, muitas vezes sem um envelhecimento saudável e vivendo em um ambiente mais suscetível à obtenção de doenças transmissíveis, como a COVID-19. Além disso, possuem diminuição do sistema imunológico e doenças crônicas não infecciosas que favorecem pior prognostico em uma possível contaminação (18). Por esses fatores de risco relacionados aos idosos e a doença em questão, a dependência de familiares e cuidadores para a realização de atividades de vida diária aumenta e tem, consequentemente, um maior tempo de convivência. Muitas vezes, ainda, a pessoa responsável pelo idoso é sobrecarregada por não conseguir contar com a ajuda de terceiros pelo isolamento social, acumulando o cuidado com a pessoa de idade e as tarefas diárias. Todos esses fatores podem gerar maiores tensões e conflitos (19).

O nível mais conhecido de violência doméstica sofrida pelos idosos é o abandono. E o medo dele, pode gerar outros problemas de saúde mental, debilitando-os ainda mais, como solidão, insônia, ansiedade, perda do apetite e depressão. Outro ponto relevante é que o distanciamento social, fundamental para a redução da transmissão do COVID-19, especialmente para as pessoas com mais de 60 anos, limita o acesso dos idosos aos serviços de saúde para o acompanhamento regular, o que pode agravar ou descompensar condições clínicas pré-existentes (20).

Além disso, o enfrentamento do isolamento social favoreceu o aumento do consumo de álcool e drogas, o que potencializa os efeitos agressivos e perda de controle sobre comportamentos (12).

\section{CONCLUSÃO}

Tal revisão integrativa apresentou que os casos de violência doméstica cresceram no Brasil durante a pandemia da COVID-19 devido aos prejuízos causados pelo isolamento social como: medo, insegurança, desemprego, necessidade de as vítimas estarem mais tempo em convívio com o agressor; circunstâncias que favorecem a violência doméstica, principalmente contra as mulheres. 
Entendendo que, embora haja aumento do índice de violência doméstica neste período atípico de pandemia, tal número está em ascensão constante, há a necessidade de fomentar o conhecimento sobre o tema, como também, ações de enfrentamento e prevenção da inserção no cenário violento.

Assim, de acordo com os estudos, os governantes possuem responsabilidade de inserir medidas de enfrentamento e prevenção contra a violência doméstica, bem como o atendimento integralizado às vítimas. As propostas de intervenção relacionadas ao tema devem ter como objetivo o fortalecimento de laços afetivos e consequentes transformações positivas nas relações familiares. Com isso, se faz necessário um trabalho multidisciplinar com as famílias por meio de atividades como: visitas domiciliares conforme necessidade; atendimento psicoterapêutico para adultos, crianças, adolescentes e idosos em sessões individuais ou em grupo em bairros que apresentem grande incidência de notificação de violência domésticas; palestras para sensibilização e comitês voltados aos profissionais das áreas de atendimento e proteção às vítimas.

A violência é um problema de saúde pública que gera às vítimas grande sofrimento, se fazendo extremamente necessário a pesquisa e estudo desta temática.

\section{REFERÊNCIAS BIBLIOGRÁFICAS}

1. Violência Intrafamiliar : Orientações para a Prática em Serviço [Internet]. Cadernos de Atenção Básica; Ministério da Saúde 2002- \&nbsp; [cited 2020 Nov 5]. Available from: http://bvsms.saude. gov.br/bvs/publicacoes/cd05_19.pdf Português. 2.Global and regional estimates of violence against women: Prevalence and health effects of intimate partner violence and non-partner sexual violence [Internet]. WHO Library Cataloguing: 2013; [cited 2020 Nov 5]. Available from: https://apps.who.int /iris/bitstream/handle/10665/85239/9789241564 625_eng.pdf;jsessionid=0EE32873F733D9C624C32 9744DBAF674? sequence=1 Inglês.

3. Gautan G, Brendan K. Domestic violence against women and the COVID-19 pandemic: What is the role of psychiatry?. International Journal of Law and Psychiatry [Internet]. 2020 Aug 01 [cited 2020 Nov 5];71(101594) DOI https://doi.org/10.1016 /j.ijlp.2020.101594. Available from: https://www.sciencedirect.com/science/article/pii /S0160252720300534?via\%3Dihub

4. Gelder N. Van, Peterman A., Potts A., O'Donnell M., Thompson K., Shah N. COVID-19: Reducing the risk of infection might increase the risk of intimate partner violence. EClinicalMedicine [Internet]. 2020 Apr 13 [cited 2020 Nov 5];21. DOI https://doi.org/10.1016/j.eclinm.2020.100348.

Available from: https://www.thelancet.com/jour nals/eclinm/article/PIIS2589-5370(20)30092 4/fulltext\#\%20

5. Protecting children during Covid-19: Resources to reduce violance and abuse [Internet].; $2020 \mathrm{Apr}$ 15. End violence against children; [cited 2020 Nov 5]; Available from: https://www.endviolence.org/protecting-children-during-covid-19 outbreak

6. Azevedo MA, editor. Violência doméstica contra crianças e adolescentes: compreensão do fenômeno no Brasil. I Jornada Internacional sobre a Infância e Violência Doméstica/Proteção e prevenção. Laboratório da Criança LACRI/IPUSP; 2012.

7. Bandeira LM. Violência de gênero: a construção de um campo teórico e de investigação. Revista Sociedade Estado. 2014

8. World Health Organization. Global and regional estimates of violence against women: prevalence and health effects of intimate partner violence and non-partner sexual violence; Genebra. 2013.

9. Ministério do Planejamento, Orçamento e Gestão. Pesquisa nacional por amostra de domicílios: síntese de indicadores 2015 [Internet]. Rio de Janeiro: Instituto Brasileiro de Geografia e Estatística; 2016 [cited 2020 Nov 5]. 109 p. Available from: : https://biblioteca.ibge.gov.br/ visualizacao/livros/liv98887.pdf

10. Ministério do Planejamento, Orçamento e Gestão. Brasil: manual de enfrentamento à violência contra a pessoa idosa. É possível prevenir. É necessário superar [Internet]. Brasília: Secretaria de Direitos Humanos da Presidência da República; 2014 [cited 2020 Nov 5]. 90 p. Available from: http://www.sdh.gov.br/assuntos/pessoaidosa/publicacoes/violencia-contra-a-pessoa-idosa 11. Ministério da Saúde. Brasil: Boletim Epidemiológico [Internet]. 2020 Apr 09 [cited 2020 
Jun 1] Available from: https://www.saude.gov.br /images/pdf/2020/April/09/be-covid-08-final2.pdf

12. Ministério da Mulher, da Família e dos Direitos Humanos, Brasil. Coronavirus: sobe o número de ligações para canal de denúncia de violência doméstica na quarentena. 2020 Apr 16.; [cited 2020 Nov 7]; Available from: https://www.gov.br/mdh/pt-br/assuntos/noticias /2020-2/marco/coronavirus-sobe-o-numero-deligacoes-para-canal-de-denuncia-de-violenciadomestica-na-quarentena

13. Secretaria de Saúde do Distrito Federal e Subsecretaria de Vigilância à Saúde. Violência interpessoal e autoprovocada em tempos de Covid-19. 2020.; [cited 2020 jul 13]; Available from: http://www.saude.df.gov.br/wp-conteudo/upload s/2018/05/INFORME-EPIDEMIOLOGICO-COVIDpublicação.pdf

14. Marques ES, Moraes CL, Hasselmann MH. Violence against woman, children and adolescents during the COVID-19 pandemic: overview, contributing factors and mitigating measures [Internet]. Cadernos de Saúde Pública; 2020. [cited 2020 Nov. 5]36. DOI http://dx.doi.org/10.1590/01

02-311x00074420. Available from: Violence against women, children, and adolescents during the COVID-19 pandemic: overview, contributing factors, and mitigating measures (scielo.br)

15. United Nations Population Fund HQ. COVID-19: um olhar para gênero: Proteção da Saúde e dos Direitos Sexuais e Reprodutivos e Promoção da Igualdade de Gênero. UNFPA [Internet]. 2020 março [cited 2020 May 5]; Available from: https://brazil.unfpa.org/sites/default/files/pubpdf/covid19_olhar_genero.pdf

16. Mazza M, Marano G, Janiri L, Sani G. Danger in Danger: Interpersonal violence during COVID-19 quarantine. Psychiatry Res. 2020;(289:113046)

17. Alcântara AO, Camarano AA, Giacomin KC. Velhas e Novas Questões. Política Nacional do Idoso. 2016;

18. Tier CG, Fontana RT, Soares NV. Refletindo sobre idosos institucionalizados. Revista Brasileira de Enfermagem. 2004;

19. Ribeiro AP, Moraes CL, Sousa ER, Giacomin KC. $O$ que fazer para cuidar das pessoas idosas e evitar as violências em época de pandemia. Política Nacional do Idoso. 2016;

20. Ong $A D$, Uchino $B N$, Wethington E. Loneliness and Healt in Older Adults. A Mini-Review and Synthesis. 2016; 